\title{
Expression profile and prognostic value of NNMT in patients with pancreatic cancer
}

\author{
Yong $\mathrm{Xu}^{1,3 *}$, Ping Liu2*, Dong-Hui Zheng ${ }^{3}$, Nan $\mathrm{Wu}^{4}$, Lun Zhu ${ }^{5}$, Changying Xing ${ }^{6}$, \\ Jin Zhu $\mathbf{u}^{1,7}$ \\ ${ }^{\mathbf{1}}$ Key Laboratory of Antibody Technique of Ministry of Health, Nanjing Medical University, Nanjing, China \\ ${ }^{2}$ Department of Pathology, Shiyan Taihe Hospital, Hubei University of Medicine, Shiyan City, China \\ ${ }^{3}$ Department of Nephrology, the Affiliated Huai'an Hospital of Xuzhou Medical College, Huai'an, China \\ ${ }^{4}$ Department of Pathology, Jinling Hospital, Nanjing, China \\ ${ }^{5}$ Department of Pathology, the Affiliated Huai'an Hospital of Xuzhou Medical College, Huai'an, China \\ ${ }^{6}$ Department of Nephrology, The First Affiliated Hospital of Nanjing Medical University, Nanjing, China \\ ${ }^{7}$ Huadong Medical Institute of Biotechniques, Nanjing, China \\ *These authors contributed equally to this work \\ Correspondence to: Jin Zhu, e-mail: zhujin1968@njmu.edu.cn \\ Changying Xing, e-mail: cyxing62@yeah.net
}

Keywords: pancreatic cancer, nicotinamide $N$-methyltransferase, clinicopathological features, overall survival

Received: November 22, 2015

Accepted: February 09, 2016

Published: March 03, 2016

\section{ABSTRACT}

The elevation of Nicotinamide $\mathrm{N}$-methyltransferase (NNMT) has been reported in pancreatic cancer tissues and cell lines, but its clinical and prognostic implications remain controversial. This study aimed at investigating the expression of NNMT in pancreatic benign and malignant tissues and the prognostic value of NNMT in pancreatic cancer. The expression of NNMT in tissue specimens of $\mathbf{2 8}$ chronic pancreatitis patients and 178 pancreatic cancer patients were assayed with immunohistochemistry on tissue microarray. The NNMT expression levels of pancreatic patients were correlated with their clinicopathological characteristics. The influences of NNMT expression and patients' clinicopathological characteristics on overall survival (OS) were analyzed. The percentage of NNMT high expression $\left(N N M T^{\mathrm{h}}\right)$ in pancreatic cancer $(55.6 \%)$ was significantly higher than those in chronic pancreatitis $(21.4 \%)$ and paracancerous tissues $(14.8 \%)(p<0.001)$. NNMT ${ }^{\mathrm{h}}$ tends to significantly correlate with unfavorable clinicopathological features such as age $>60$ years old $(p=0.014)$, tumor diameter $>4 \mathrm{~cm}(p<0.001)$, TNM stage III or IV $(p<0.001)$ and poor tumor differentiation $(p=0.004)$. The median OS of patients with $N_{N M T}{ }^{\mathrm{h}}$ and $\mathrm{NNMT}^{\mathrm{l}}$ were 7.0 months $(95 \%$ CI: 5.275-8.725) and 11.5 months (95\% CI: 9.759-13.241) respectively $(p=0.005)$. On multivariate analysis, NNMT' (hazards ratio [HR]: $0.399 ; 95 \%$ CI: $0.284-0.560$; $p<0.001$ ), absence of neurological involvement (HR: $0.651 ; 95 \%$ CI: $0.421-0.947$; $p=0.041$ ), TNM stage I or II (HR: $0.506 ; 95 \%$ CI: $0.299-0.719 ; p=0.015$ ) and well tumor differentiation (HR: $0.592 ; 95 \% \mathrm{CI}$ : $0.319-0.894 ; p=0.044$ ) were significant favorable prognostic factors of OS. In conclusion, NNMT is upregulated in pancreatic cancer, correlates with unfavorable clinicopathological features and may serve as an independent prognosticator of patients' survival.

\section{INTRODUCTION}

Pancreatic cancer remains one of the deadliest cancers worldwide, with the 5 -year survival rate being only $7 \%[1,2]$ and the median over survival (OS) only 6 months
[3]. Due to lack of effective means for early diagnosis, most patients are diagnosed at an advanced stage that is no longer suitable for curative surgical resection. Therefore, there remains an urgent need to seek potential diagnostic biomarkers and therapeutic targets of pancreatic cancer. 
Nicotinamide N-methyltransferase (NNMT) is the only known enzyme in the human body that converts nicotinamide into 1-methylnicotinamide (NMN) [4] and is involved in the biotranformation of many drugs and xenobiotics [5]. Growing evidence shows that NNMT is aberrantly expressed in and is associated with the malignancy degree of many cancers such as bladder cancer [6], lung cancer [7] colorectal cancer [8], gastric cancer [9] and hepatocellular carcinoma [10]. As for NNMT expression in pancreatic cancer, previous studies have shown that NNMT is upregulated in pancreatic cancer tissue and cell lines as well as in the pancreatic juice obtained from pancreatic cancer patients [11-13]. However, there are some controversies regarding the role of NNMT in pancreatic cancer. Bi HC, et, al. [14] revealed that the sizes of xenograft tumors formed by PANC-1 cells (a human pancreatic cancer cell line) were inversely correlated with the levels of NNMT expression, whereas Yu T, et, al. reported that NNMT silencing and overexpression reduced and enhanced the malignancy of PANC-1 cells respectively [15]. In a small number of pancreatic cancer patients $(n=22)$, no significant association was found between the patients' overall survival and NNMT expression in the cancer specimens [14]. Therefore, the role of NNMT expression level in pancreatic cancer and its clinical significance remain elusive.

In this study, we assayed the expression levels of NNMT in tissues of chronic pancreatitis, pancreatic cancer and paracancerous specimen, and then investigated the relationship between NNMT expression level and patients' clinicopathological characteristics as well as patients' OS.

\section{RESULTS}

\section{NNMT expression in chronic pancreatitis, pancreatic cancer and paracancerous tissues}

At last, a total of 178 patients with pancreatic cancer and 28 patients with chronic pancreatitis were included. Tissue samples of these patients were obtained from the Department of Pathology, Jinling Hospital (Nanjing, China), including 28 chronic pancreatitis, 178 pancreatic cancer tissues and 61 paracancerous tissues. Immunohistochemistry was performed to determine NNMT expression in these 267 samples. Representative immunohistochemically stained sections of chronic pancreatitis, paracancerous tissues and pancreatic cancer are shown in Figure 1.

Using the X-tile software program for TMA data analysis (http://www. tissuearray.org/rimmlab), we identified 110 as the significant cutoff point in terms of low and high expression of NNMT in all tissue samples, i.e., score $0-109$ was considered as low expression $\left(\mathrm{NNMT}^{\mathrm{l}}\right)$ whereas 110-300 as high expression $\left(\mathrm{NNMT}^{\mathrm{h}}\right)$.
As showed in Table 1, the percentage of pancreatic cancer samples with $\mathrm{NNMT}^{\mathrm{h}}$ was 55.6\% (99/178), significantly higher than that of paracancerous tissues $(21.4 \%)$ and chronic pancreatitis $(14.8 \%)(p<0.001)$, indicating that in general, NNMT is upregulated in pancreatic cancer.

\section{Association of NNMT expression with clinicopathological characteristics in patients with pancreatic cancer}

Table 2 lists the correlation between $\mathrm{NNMT}^{\mathrm{h}}$ and the clinicopathological parameters of pancreatic cancer patients. As shown in this table, $\mathrm{NNMT}^{\mathrm{h}}$ was significantly correlated with older age $(p=0.014)$, larger tumor size $(p<0.001)$, more advanced TNM stage $(p<0.001)$, moderate-to-poor differentiation $(p=0.004)$, and higher CA19-9 level $(p=0.005)$. These results indicate that $\mathrm{NNMT}^{\mathrm{h}}$ tends to correlate with unfavorable clinicopathological features in pancreatic cancer patients compared with NNMT ${ }^{1}$.

\section{The prognostic value of NNMT expression in pancreatic cancer}

By the last follow-up, only 9 patients were still alive. The median OS of patients with $\mathrm{NNMT}^{\mathrm{h}}$ and $\mathrm{NNMT}^{\mathrm{l}}$ were 7.0 months (95\% CI: 5.275-8.725) and 11.5 months (95\% CI: 9.759-13.241) respectively ( $p=0.005)$ (Table 3 and Figure 2). Besides NNMT expression level, other factors that impact the median OS on univariate analyses include age $(p=0.011)$, tumor diameter $(p=0.027)$, neurological involvement $(p=0.033)$, TNM stage $(p=0.001)$, distance metastasis $(p<0.001)$ and tumor differentiation $(p=0.021)$ (Table 3$)$.

On multivariate analysis, $\mathrm{NNMT}^{1}$ (hazards ratio [HR]: 0.399; 95\% CI: 0.284-0.560; $p<0.001)$, absence of neurological involvement (HR: $0.651 ; 95 \%$ CI: $0.421-$ $0.947 ; p=0.041$ ), TNM stage I or II (HR: $0.506 ; 95 \% \mathrm{CI}$ : $0.299-0.719 ; p=0.015$ ) and well tumor differentiation (HR: 0.592; 95\% CI: $0.319-0.894 ; p=0.044$ ) were significant favorable prognostic factors of OS compared with $\mathrm{NNMT}^{\mathrm{h}}$, presence of neurological involvement, TNM stage III or IV and poor differentiation, respectively (Table 3).

\section{DISCUSSION}

In this study, we showed that the percentage of $\mathrm{NNMT}^{\mathrm{h}}$ in pancreatic cancer was significantly higher than those in pancreatic benign tissues, in line with the previous findings that NNMT is elevated in pancreatic cancer $[11,12]$. Moreover, we for the first time revealed that $\mathrm{NNMT}^{\mathrm{h}}$ tends to correlate with unfavorable clinicopathological features in pancreatic cancer patients and that $\mathrm{NNMT}^{\mathrm{h}}$ is an independent unfavorable prognosticator of OS, suggesting an oncogenic role of NNMT in this cancer. 
Table 1: NNMT gene expression in pancreatic benign and malignant tissues

\begin{tabular}{|l|c|c|c|c|}
\hline \multicolumn{1}{c}{ Tissues } & $n$ & NNMT $^{\text {h }}(\%)$ & Pearson $\chi^{2}$ & \multicolumn{1}{c|}{ P } \\
\hline Chronic pancreatitis & 28 & $6(21.4)$ & 11.317 & $0.001^{\dagger}$ \\
\hline Paracancerous tissues & 61 & $9(14.8)$ & 30.629 & $<0.001^{*}$ \\
\hline Pancreatic cancer & 178 & $99(55.6)$ & 26.134 & $<0.001^{\#}$ \\
\hline
\end{tabular}

$\mathrm{NNMT}^{\mathrm{h}}$ : NNMT high expression; $\uparrow:$ Chronic pancreatitis versus pancreatic cancer;

*: Paracancerous tissues versus pancreatic cancer; \#: Among the three groups.

A



Figure 1: Representative presentation of NNMT protein expression in benign and malignant pancreatic tissues on tissue microarray sections by IHC. Row 1 and 2 are NNMT staining observed at low $(\times 40$; bar $=500 \mu \mathrm{m})$ and high $(\times 400$; bar $=50 \mu \mathrm{m}$ ) magnification respectively. Column (A) Chronic pancreatitis with low expression of NNMT; Column (B) Paracancerous tissue with low NNMT expression; Column $(\mathbf{C}-\mathbf{E})$ show high NNMT expression in pancreatic cancer with well, moderate and poor differentiation respectively. IHC: immunohistochemistry; NNMT: Nicotinamide N-methyltransferase.

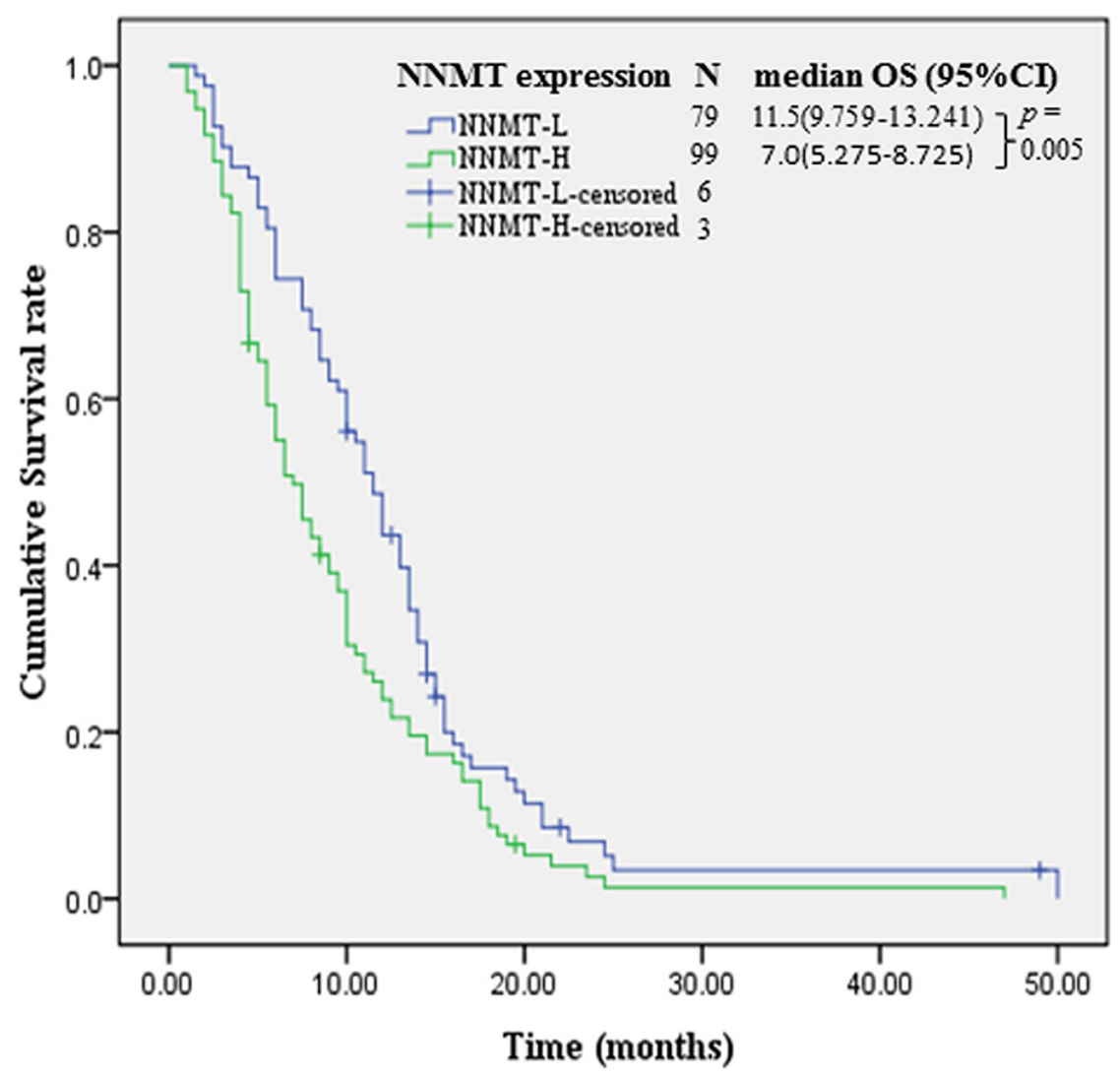

Figure 2: Kaplan-Meier survival curves of patients by NNMT expression levels. NNMT: Nicotinamide N-methyltransferase. $\mathrm{NNMT}^{\mathrm{h}}$ : NNMT high expression; $\mathrm{NNMT}^{\mathrm{l}}$ : NNMT low expression. 
Table 2: Association of NNMT expression with clinicopathological characteristics in patients with pancreatic cancer

\begin{tabular}{|c|c|c|c|c|}
\hline Patients' characteristics & & NNMT $^{\mathrm{h}}(\%)$ & Pearson $\chi^{2}$ & $\boldsymbol{P}$ \\
\hline Total & 178 & $99(55.6)$ & & \\
\hline \multirow{2}{*}{ Age (years) } & $\leq 60(n=83)$ & $38(45.8)$ & \multirow{2}{*}{6.094} & \multirow{2}{*}{0.014} \\
\hline & $>60(n=95)$ & $61(64.2)$ & & \\
\hline \multirow{2}{*}{ Gender } & Male $(n=104)$ & $57(54.8)$ & \multirow{2}{*}{0.067} & \multirow{2}{*}{0.796} \\
\hline & Female $(n=74)$ & $42(56.8)$ & & \\
\hline \multirow{2}{*}{ Diameter $(\mathrm{cm})$} & $<4(n=107)$ & $48(44.9)$ & \multirow{2}{*}{12.578} & \multirow{2}{*}{$<0.001$} \\
\hline & $\geq 4(n=71)$ & $51(71.8)$ & & \\
\hline \multirow{2}{*}{$\begin{array}{l}\text { Neurological } \\
\text { involvement }\end{array}$} & Absent $(n=99)$ & $57(57.6)$ & \multirow{2}{*}{0.346} & \multirow{2}{*}{0.556} \\
\hline & Present $(n=79)$ & $42(53.2)$ & & \\
\hline \multirow{2}{*}{ TNM stage } & I or II $(n=123)$ & $56(45.5)$ & \multirow{2}{*}{16.417} & \multirow{2}{*}{$<0.001$} \\
\hline & III or IV $(n=55)$ & $43(78.2)$ & & \\
\hline \multirow{2}{*}{$\mathrm{T}$} & T1 or T2 $(n=94)$ & $52(55.3)$ & \multirow{2}{*}{0.007} & \multirow{2}{*}{0.932} \\
\hline & T3 or T4 $(n=84)$ & $47(56.0)$ & & \\
\hline \multirow{2}{*}{$\mathrm{N}$} & N0 $(n=126)$ & $59(46.8)$ & \multirow{2}{*}{13.508} & \multirow{2}{*}{$<0.001$} \\
\hline & $\mathrm{N} 1(n=52)$ & $40(76.9)$ & & \\
\hline \multirow{2}{*}{ M } & M0 $(n=140)$ & $67(47.9)$ & \multirow{2}{*}{16.001} & \multirow{2}{*}{$<0.001$} \\
\hline & M1 $(n=38)$ & $32(84.2)$ & & \\
\hline \multirow{3}{*}{ Differentiation } & Well $(n=17)$ & $3(17.6)$ & \multirow{3}{*}{10.997} & \multirow{3}{*}{0.004} \\
\hline & Moderate $(n=103)$ & $61(59.2)$ & & \\
\hline & Poor $(n=58)$ & $35(60.3)$ & & \\
\hline \multirow{2}{*}{$\begin{array}{l}\text { Preoperative } \\
\text { CEA (ng/ml) }\end{array}$} & $\leq 5(n=106)$ & $59(55.7)$ & \multirow{2}{*}{0.000} & \multirow{2}{*}{0.989} \\
\hline & $>5(n=72)$ & $40(55.6)$ & & \\
\hline \multirow{2}{*}{ Preoperative CA19-9 (U/mL) } & $\leq 34(n=74)$ & $32(43.2)$ & \multirow{2}{*}{7.857} & \multirow{2}{*}{0.005} \\
\hline & $>34(n=104)$ & 67 (64.4) & & \\
\hline
\end{tabular}

NNMT $^{\text {h }}$ NNMT high expression; CEA: carcinoembryonic antigen.

Previous studies have reported an increased incidence of pancreatic cancer in patients with a history of chronic pancreatitis [16]. Our study shows that the percentage of $\mathrm{NNMT}^{\mathrm{h}}$ in pancreatic cancer was significant higher than that in chronic pancreatitis $(55.6 \%$ vs $21.4 \%, p<0.001)$, indicating that NNMT expression level has the potential to distinguish pancreatic benign from malignant lesions.

To date, there are only very few reports $[14,15]$ suggesting the possible roles of NNMT in pancreatic cancer and the results are controversial. Bi HC, et, al. [14] reported that the sizes of xenograft tumors were inversely correlated with NNMT expression, suggesting an oncosuppressive role of NNMT in pancreatic cancer. Yu T, et, al. [15], on the contrary, reported that NNMT silencing and overexpression reduced and enhanced the malignancy of pancreatic cancer cells respectively, suggesting an oncogenic role. Our results showed that NNMT ${ }^{\mathrm{h}}$ tends to correlate with unfavorable clinicopathological features in pancreatic cancer patients, in support of its oncogenic role, although the underlying mechanisms remain elusive. $\mathrm{Bi}$ $\mathrm{HC}$, et, al. [14] had investigated the prognostic value of NNMT expression level in a small number of pancreatic cancer patients $(n=22)$ but failed to find any positive result, possibly due to the small cohort size. Our study for the first time revealed that compared with $\mathrm{NNMT}^{\mathrm{h}}$, $\mathrm{NNMT}^{1}$ is an independent and significant favorable prognosticator of patients with pancreatic cancer.

As we know, the dismal prognosis of pancreatic cancer is at least partially attributable to the lack of effective and convenient means of early diagnosis. During the past decades, many potential biomarkers have been tested [17] but only CA 19-9 shows great promise $[18,19]$. Yet, CA 19-9 is not a satisfactory biomarker because it is not specific enough [20] and about $10 \%$ of pancreatic cancer patients present negative CA 19-9 value even in advanced stages of the disease [21]. Therefore, there remains an urgent need to develop novel biomarkers. Our study adds a new member to the molecular candidates 
Table 3: Univariate and multivariate survival analyses by clinicopathological characteristics and NNMT expression in patients with pancreatic cancer

\begin{tabular}{|c|c|c|c|c|c|}
\hline & & \multicolumn{2}{|l|}{ Univariate analysis } & \multicolumn{2}{|l|}{ Multivariate analysis } \\
\hline & & mOS (95\% CI), months & $P$ value & $\begin{array}{l}\text { Hazard ratio } \\
(95 \% \mathrm{CI})\end{array}$ & $P$ value \\
\hline Total & 178 & \multicolumn{4}{|l|}{$9.0(6.761-11.239)$} \\
\hline \multirow{2}{*}{$\begin{array}{l}\text { Age } \\
\text { (years) }\end{array}$} & $\leq 60(n=83)$ & $11.0(9.896-12.104)$ & \multirow{2}{*}{0.011} & $0.870(0.632-1.197)$ & \multirow{2}{*}{0.392} \\
\hline & $>60(n=95)$ & $6.5(5.555-7.445)$ & & 1 & \\
\hline \multirow{2}{*}{ Gender } & Male $(n=104)$ & $9.0(6.671-11.239)$ & \multirow{2}{*}{0.312} & & \\
\hline & Female $(n=74)$ & $9.0(7.679-10.321)$ & & & \\
\hline \multirow{2}{*}{$\begin{array}{l}\text { Diameter } \\
(\mathrm{cm})\end{array}$} & $<4(n=107)$ & $11.2(10.019-12.412)$ & \multirow{2}{*}{0.027} & $0.759(0.525-1.116)$ & \multirow{2}{*}{0.103} \\
\hline & $\geq 4(n=71)$ & $7.4(6.169-9.007)$ & & 1 & \\
\hline \multirow{2}{*}{$\begin{array}{l}\text { Neurological } \\
\text { Involvement }\end{array}$} & Absent $(n=99)$ & $10.7(9.343-12.002)$ & \multirow{2}{*}{0.033} & $0.651(0.421-0.947)$ & \multirow{2}{*}{0.041} \\
\hline & Present $(n=79)$ & $7.2(6.024-9.121)$ & & 1 & \\
\hline \multirow{2}{*}{ TNM stage } & I or II $(n=123)$ & $10.0(8.470-11.530)$ & \multirow{2}{*}{0.001} & $0.506(0.299-0.719)$ & \multirow{2}{*}{0.015} \\
\hline & III or IV $(n=55)$ & $7.5(5.156-9.844)$ & & 1 & \\
\hline \multirow{2}{*}{$\mathrm{T}$} & T1 or T2 $(n=94)$ & $10.6(8.829-11.771)$ & \multirow{2}{*}{0.47} & & \\
\hline & T3 or T4 $(n=84)$ & $8.5(6.742-10.258)$ & & & \\
\hline \multirow{2}{*}{$\mathrm{N}$} & N0 $(n=126)$ & $10.4(8.508-11.792)$ & \multirow{2}{*}{0.127} & & \\
\hline & $\mathrm{N} 1(n=52)$ & $8.0(6.459-9.541)$ & & & \\
\hline \multirow{2}{*}{ M } & $\operatorname{M0}(n=140)$ & $9.7(8.733-11.267)$ & \multirow{2}{*}{$<0.001$} & $0.706(0.485-1.056)$ & \multirow{2}{*}{0.092} \\
\hline & M1 $(n=38)$ & $6.8(4.558-8.442)$ & & 1 & \\
\hline \multirow{3}{*}{ Differentiation } & Well $(n=27)$ & $11.4(8.966-14.434)$ & \multirow{3}{*}{0.021} & $0.592(0.319-0.894)$ & \multirow{3}{*}{0.044} \\
\hline & Moderate $(n=69)$ & $9.5(7.447-11.553)$ & & $0.832(0.611-1.121)$ & \\
\hline & Poor $(n=82)$ & $7.2(5.601-8.689)$ & & 1 & \\
\hline \multirow{2}{*}{$\begin{array}{l}\mathrm{CEA}^{\#} \\
(\mathrm{ng} / \mathrm{ml})\end{array}$} & $\leq 5(n=106)$ & $9.8(6.977-13.023)$ & \multirow{2}{*}{0.373} & & \\
\hline & $>5(n=72)$ & $8.5(6.681-10.319)$ & & & \\
\hline \multirow{2}{*}{$\begin{array}{l}\text { CA19-9" } \\
(\mathrm{U} / \mathrm{mL})\end{array}$} & $\leq 34(n=34)$ & $10.0(8.314-11.686)$ & \multirow{2}{*}{0.326} & & \\
\hline & $>34(n=144)$ & $7.5(5.385-9.615)$ & & & \\
\hline \multirow{2}{*}{ NNMT expression } & $\operatorname{NNMT}^{\mathrm{h}}(n=99)$ & $7.0(5.275-8.725)$ & 0005 & 1 & \\
\hline & $\operatorname{NNMT}^{1}(n=79)$ & $11.5(9.759-13.241)$ & 0.000 & $0.399(0.284-0.560)$ & .001 \\
\hline
\end{tabular}

\#: Preoperative; mOS: median overall survival; CEA: carcinoembryonic antigen.

holding potentials to be developed as diagnostic and prognostic biomarkers of pancreatic cancer.

Although patients with TNM stage III and IV pancreatic cancer are unsuitable for surgical resection in mainstream opinions [22], some stage III or IV patients in our hospital indeed received surgical treatment for palliative purposes upon the request of patients and after the careful evaluation by the institutional expert team. So, there were 55 patients with stage III or IV pancreatic cancer in our study whose participation enabled us to investigate the expression and prognostic value of NNMT in this subset of patients. One limitation of our study is that we did not set foot in the mechanisms underlying NNMT elevation and the mechanisms underlying its prognostic value in pancreatic cancer, which should be addressed by future studies.

In conclusion, NNMT is upregulated in pancreatic cancer, correlates with unfavorable clinicopathological features and holds the potential to be developed as aprognosticator of patients' survival. The results of our study require further validation in a larger patient cohort and the mechanisms underlying these results merit further investigation to explore the possibilities of NNMT as a therapeutic target of pancreatic cancer. 


\section{MATERIALS AND METHODS}

\section{Human tissue specimens and patient clinical information}

This study was approved by the Human Research Ethics Committee of Jinling Hospital (Nanjing, China). Treatment-naive patients with pancreatic cancer who received pancreatectomy as the initial treatment at Jinling Hospital (Nanjing, China) from Jan 1st, 2010 to Dec 31st, 2013 were included in this study. The diagnoses of pancreatic cancer were confirmed by postoperative pathological results. Patients with chronic pancreatitis confirmed by fine needle aspiration biopsy during the same period were also included.

Clinical characteristics of cancer patients were extracted from their medical record, including age, gender, tumor diameter, neurological involvement, TNM stage, differentiation, preoperative serum carcinoembryonic antigen (CEA) and carbohydrate antigen 19-9 (CA19-9) levels. Patients with pancreatic cancer were followed up and the OS were calculated from the date of surgical treatment to the date of death or last follow-up.

\section{Immunohistochemistry analysis (IHC) on paraffin embedded tissue}

The tissue microarray block was cut into $4-\mu \mathrm{m}$ sections and immunohistochemical staining was performed as previously described [23]. Briefly, the sections were first deparaffinized and hydrated. After antigen retrieval with $0.01 \mathrm{M}$ citrate buffer $\mathrm{pH} 6.0$ and microwave heat induction, the sections were treated with $3 \%$ hydrogen peroxide for $10 \mathrm{~min}$ to block the endogenous peroxidase activity. NNMT was detected by mouse monoclonal anti-human NNMT antibody (dilution 1:150) (Novus Biologicals, USA, NBP2-00537). After secondary antibody staining, diaminobenzidine (DAB) was used as the chromogen for $3 \mathrm{~min}$, and then the nuclei were counterstained with hematoxylin. Staining results were evaluated independently by two pathologists without prior knowledge of clinicopathologic data.

The expression of NNMT was scored using the semi-quantitative $\mathrm{H}$-score method, which takes into account both the staining intensity and the percentage of cells at that intensity [24]. For each of the samples, the staining intensity was scored as 0 (no staining), $1+$ (weak staining), $2+$ (moderate staining), or $3+$ (intense staining). Then the percentage of cells stained at the respective intensity was determined and multiplied by the intensity score to yield an intensity percentage score. The final staining scores were then calculated from the sum of the four intensity percentage scores. Therefore, the staining score had a minimum value of 0 (no staining) and a maximum value of $300(100 \%$ of cells with $3+$ staining intensity).

\section{Statistical analysis}

The continuous NNMT expression data from IHC were converted into dichotic data (low vs high) using specific cutoff values, which were selected to be significant in terms of OS using the X-tile software program (The Rimm Lab at Yale University; http://www.tissuearray.org/ rimmlab) [25].

Student $t$ test and Pearson $\chi^{2}$ test were used to determine the statistical significance of differences between comparison groups. Median OS was calculated using the Kaplan-Meier method and compared by the $\log$-rank test. Variables with $P$-value $<0.20$ on univariate analyses were included in multivariate analysis (Cox proportional hazards model). $P$-value less than 0.05 was considered statistically significant. All statistical analyses were performed using SPSS 20.0 statistical software package (SPSS Inc., Chicago, IL).

\section{ACKNOWLEDGMENTS AND FUNDING}

This study was funded by The National Natural Science Foundation of China (81270817) and The Scientific Research Foundation of Huai'an (HA2014003; HAP201119).

\section{CONFLICTS OF INTEREST}

The authors declare no conflicts interests.

\section{REFERENCES}

1. Siegel R, Miller KD, Jemal A. Cancer statistics, 2015. CA Cancer J Clin. 2015; 65:5-29.

2. Muniraj T, Jamidar PA, Aslanian HR. Pancreatic cancer: a comprehensive review and update. Dis Mon. 2013; 59:368-402.

3. Gong Z, Holly EA, Bracci PM. Survival in populationbased pancreatic cancer patients: San Francisco Bay area, 1995-1999. Am J Epidemiol. 2011; 174:1373-1381.

4. Alston T, Abeles R. Substrate specificity of nicotinamide methyltransferase isolated from porcine liver. Arch Biochem Biophys. 1988; 260:601-608.

5. Aksoy S, Szumlanski CL, Weinshilboum RM. Human liver nicotinamide N-methyltransferase cDNA cloning, expression, and biochemical characterization. J Biol Chem. 1994; 269:14835-14840.

6. Wu Y, Siadaty MS, Berens ME, Hampton GM, Theodorescu D. Overlapping gene expression profiles of cell migration and tumor invasion in human bladder cancer identify metallothionein $1 \mathrm{E}$ and nicotinamide $\mathrm{N}$-methyltransferase as novel regulators of cell migration. Oncogene. 2008; 27:6679-6689.

7. Sartini D, Morganti S, Guidi E, Rubini C, Zizzi A, Giuliante R, Pozzi V, Emanuelli M. Nicotinamide $\mathrm{N}$-methyltransferase in non-small cell lung cancer: Promising results for targeted anti-cancer therapy. Cell Biochem Biophys. 2013; 67:865-873. 
8. Roessler M, Rollinger W, Palme S, Hagmann ML, Berndt P, Engel AM, Schneidinger B, Pfeffer M, Andres H, Karl J, Bodenmüller H, Rüschoff J, Henkel T, et al. Identification of nicotinamide $\mathrm{N}$-methyltransferase as a novel serum tumour marker for colorectal cancer. Clin Cancer Res. 2005; 11:6550-6557.

9. Jang JS, Cho HY, Lee YJ, Ha WS, Kim HW: The differential proteome profile of stomach cancer: Identification of the biomarker candidates. Oncol Res 2004; 14:491-499.

10. Kim J, Hong SJ, Lim EK, Yu YS, Kim SW, Roh JH, Do IG, Joh JW, Kim DS. Expression of nicotinamide $\mathrm{N}$-methyltransferase in hepatocellular carcinoma is associated with poor prognosis. J Exp Clin Cancer Res. 2009; 28:20.

11. Iacobuzio-Donahue CA, Maitra A, Shen-Ong GL, van Heek T, Ashfaq R, Meyer R, Walter K, Berg K, Hollingsworth MA, Cameron JL, Yeo CJ, Kern SE, Goggins M, et al. Discovery of Novel Tumor Markers of Pancreatic Cancer using Global Gene Expression Technology, Am J Pathol. 2002; 160:1239-1249.

12. Logsdon CD, Simeone DM, Binkley C, Arumugam T, Greenson JK, Giordano TJ, Misek DE, Kuick R, Hanash S. Molecular Profiling of Pancreatic Adenocarcinoma and Chronic Pancreatiti Identifies Multiple Genes Differentially Regulated in Pancreatic Cancer. Cancer Research. 2003; 63:2649-2657.

13. Rogers CD, Fukushima N, Sato N, Shi C, Prasad N, Hustinx SR, Matsubayashi H, Canto M, Eshleman JR, Hruban RH, Goggins M. Differentiating pancreatic lesions by microarray and qPCR analysis of pancreatic juice rnas. Cancer Biol Ther. 2006; 5:1383-1389.

14. Bi HC, Pan YZ, Qiu JX, Krausz KW, Li F, Johnson CH, Jiang CT, Gonzalez FJ, Yu AM. N-methylnicotinamide and nicotinamide $\mathrm{N}$-methyltransferase are associated with microRNA-1291-altered pancreatic carcinoma cell metabolome and suppressed tumorigenesis. Carcinogenesis. 2014; 35:2264-72.

15. Yu T, Wang YT, Chen $\mathrm{P}, \mathrm{Li} \mathrm{YH}$, Chen YX, Zeng H, Yu AM, Huang M, Bi HC. Effects of nicotinamide N-methyltransferase on PANC-1 cells proliferation, metastatic potential and survival under metabolic stress. Cell Physiol Biochem. 2015; 35:710-21.
16. Batty GD, Kivimaki M, Morrison D, Huxley R, Smith GD, Clarke R, Marmot MG, Shipley MJ. Risk factors for pancreatic cancer mortality: extended follow-up of the original Whitehall Study. Cancer Epidemiol Biomarkers Prev. 2009; 18:673-675.

17. Harsha HC, Kandasamy K, Ranganathan P, Rani S, Ramabadran S, Gollapudi S, Balakrishnan L, Dwivedi SB, Telikicherla D, Selvan LD, Goel R, Mathivanan S, Marimuthu A, et al. A compendium of potential biomarkers of pancreatic cancer. PLoS Med. 2009; 6:e1000046.

18. Steinberg W. The clinical utility of the CA 19-9 tumorassociated antigen. Am J Gastroenterol. 1990; 85:350-5.

19. Poruk KE, Gay DZ, Brown K, Mulvihill JD, Boucher KM, Scaife CL, Firpo MA, Mulvihill SJ. The clinical utility of CA 19-9 in pancreatic adenocarcinoma: diagnostic and prognostic updates. Curr Mol Med. 2013; 13:340-51.

20. Ballehaninna UK, Chamberlain RS. The clinical utility of serum CA 19-9 in the diagnosis, prognosis and management of pancreatic adenocarcinoma: An evidence based appraisal. J Gastrointest Oncol. 2012; 3:105-119.

21. Frebourg T, Bercoff E, Manchon N, Senant J, Basuyau JP, Breton P, Janvresse A, Brunelle P, Bourreille J. The evaluation of CA 19-9 antigen level in the early detection of pancreatic cancer. A prospective study of 866 patients. Cancer. 1988; 62:2287-2290.

22. Kanji ZS, Gallinger S. Diagnosis and management of pancreatic cancer. CMAJ. 2013; 185:1219-26.

23. Sun RW, Wang XD, Zhu HJ, Mei HJ, Wang W, Zhang S, Huang JF. Prognostic value of LAMP3 and TP53 overexpression in benign and malignant gastrointestinal tissues. Oncotarget. 2014; 5:12398-12409. doi: 10.18632/ oncotarget. 2643.

24. Detre S, Saclani Jotti G, Dowsett M. A “quickscore" method for immunohistochemical semiquantitation: validation for oestrogen receptor in breast carcinomas. J Clin Pathol. 1995; 48:876-878.

25. Ni S, Xu L, Huang J, Feng J, Zhu H, Wang G, Wang X. Increased ZO-1 expression predicts valuable prognosis in non-small cell lung cancer. Int J Clin Exp Pathol. 2013; 6:2887-2895. 